\title{
Complex Geodesics of the Minimal Ball in $\mathbb{C}^{n}$
}

\author{
PETER PFLUG - EL HASSAN YOUSSFI
}

\begin{abstract}
In this note we give a characterization of the complex geodesics of the minimal ball in $\mathbb{C}^{n}$. This answers a question posed by Jarnicki and Pflug (cf. [JP], Example 8.3.10)
\end{abstract}

Mathematics Subject Classification (2000): 32F45.

\section{1. - Introduction}

$\mathbb{B}_{*}:=\left\{z \in \mathbb{C}^{n}: N_{*}(z):=\sqrt{|z|^{2}+|z \bullet z|}<1\right\}$, where $z \bullet z:=z_{1}^{2}+\cdots+z_{n}^{2}$,

be the so-called minimal ball in $\mathbb{C}^{n}$ (see [HP]). Recall that $N_{*}$ is a norm on $\mathbb{C}^{n}$. This ball has been shown to be of interest in several recent works ([K], [MY], [OY], [OPY], [Z]). In particular, it is a non Lu Qi-Keng domain for $n \geq 4$ (see $[\mathrm{PY}]$ ), and it is neither homogeneous nor Reinhardt. Furthermore, the boundary $\partial \mathbb{B}_{*}$ of $\mathbb{B}_{*}$ is smooth only outside of the set $\left\{z \in \mathbb{C}^{n}: z \bullet z=0\right\}$ and smooth boundary points are strictly pseudoconvex.

Denote by $\Delta$ the unit disc in $\mathbb{C}$. The first goal of this paper is to establish an explicit necessary form for a mapping $\varphi: \Delta \longrightarrow \mathbb{B}_{*}$ to be a complex geodesic (for definitions and properties see [JP]). Observe that complex geodesics give important information about the complex geometry of the domain. The first main result is:

Theorem A. A complex geodesic $\varphi=\left(\varphi_{1}, \ldots, \varphi_{n}\right): \Delta \longrightarrow \mathbb{B}_{*}$ is of the form

$$
\varphi_{j}(\lambda)=a_{j}\left(\frac{\lambda-\alpha_{j 1}}{1-\bar{\alpha}_{j 1} \lambda}\right)^{k_{j 1}}\left(\frac{\lambda-\alpha_{j 2}}{1-\bar{\alpha}_{j 2} \lambda}\right)^{k_{j 2}} \frac{\left(1-\bar{\alpha}_{j 1} \lambda\right)\left(1-\bar{\alpha}_{j 2} \lambda\right)}{(1-\bar{\alpha} \lambda)^{2}},
$$

This paper was started during the visit of the first author at the Universite de Provence in 2003 and continued during the visit of the second author at the university of Oldenburg in 2003. The authors were supported by the Université de Provence and the DFG. We like to thank these institutions for their support.

Pervenuto alla Redazione il 28 ottobre 2003. 
where $a_{j} \in \mathbb{C}, \alpha \in \Delta, k_{j s} \in\{0,1\}, \alpha_{j s} \in \bar{\Delta}$ and $\alpha_{j s} \in \Delta$ whenever $k_{j s}=1$, $s=1,2$, and $\left(k_{j 1}, k_{j 2}\right) \neq(1,1), j=1, \ldots, n$. Moreover, a geodesic through two different points is uniquely determined (up to automorphisms of $\Delta$ ). In addition, $\varphi \bullet \varphi$ has the form

$$
\varphi \bullet \varphi=b\left(\frac{\lambda-\beta_{1}}{1-\bar{\beta}_{1} \lambda}\right)^{k_{1}}\left(\frac{\lambda-\beta_{2}}{1-\bar{\beta}_{2} \lambda}\right)^{k_{2}} \frac{\left(1-\bar{\beta}_{1} \lambda\right)^{2}\left(1-\bar{\beta}_{2} \lambda\right)^{2}}{(1-\bar{\alpha} \lambda)^{4}}
$$

where $b \in \mathbb{C}$ and $k_{s} \in\{0,1\}, \beta_{s} \in \bar{\Delta}$ for $s=1,2$ with the understanding that $\beta_{s} \in \Delta$ whenever $k_{s}=1$. In particular, if $\varphi \bullet \varphi \not \equiv 0$, then $\varphi \bullet \varphi$ has at most two zeros in $\Delta$.

A second goal is to give necessary and sufficient conditions for a mapping of the form (1.1) and (1.2) to be a complex geodesic from $\Delta$ into $\mathbb{B}_{*}$. See Proposition 3.3 below.

\section{2. - Proof of Theorem A}

For $w \in \mathbb{C}^{n}$, let

$$
\widehat{q}(w):=\max \left\{\Re(z \bullet w): z \in \partial \mathbb{B}_{*}\right\} .
$$

Then by Corollary 8.2.8 in [JP] we have

LeMma 2.1. A holomorphic mapping $\varphi: \Delta \longrightarrow \mathbb{B}_{*}$ is a complex geodesic if and only if its boundary values $\varphi^{*}(\lambda)$ belong to $\partial \mathbb{B}_{*}$ for almost all $\lambda \in \partial \Delta$ and $\varphi$ is stationary, i.e. there is a holomorphic mapping $h: \Delta \longrightarrow \mathbb{C}^{n}$ with components in the Hardy space $H^{1}(\Delta)$ such that

$$
\Re\left(\varphi^{*}(\lambda) \bullet h^{*}(\lambda)\right)=\widehat{q}\left(\frac{h^{*}(\lambda)}{\lambda}\right),
$$

for almost all $\lambda \in \partial \Delta$.

In order to describe $h^{*}(\lambda) / \lambda, \lambda \in \partial \Delta$, we need the following lemma.

LeMma 2.2. If $w \in \mathbb{C}^{n} \backslash\{0\}$ and $z \in \partial \mathbb{B}_{*}$ satisfy

$$
\Re(w \bullet z)=\widehat{q}(w),
$$

then there are numbers $\varrho=\varrho(z)>0$ and $\eta=\eta(z) \in \varrho(z) \bar{\Delta}$ such that

$$
\begin{array}{ll}
w=\varrho\left[\bar{z}+\frac{\overline{z \bullet z}}{|z \bullet z|} z\right], & \text { if } z \bullet z \neq 0, \\
w=\varrho \bar{z}+\eta z, & \text { if } z \bullet z=0
\end{array}
$$


Proof. Suppose that $z, w$ are as in the hypothesis of the lemma. By (2.1) we see that

$$
\Re((\zeta-z) \bullet w)<0 \quad \text { for all } \zeta \in \mathbb{B}_{*} .
$$

If $z \bullet z \neq 0$, then $z$ is a smooth boundary point of $\mathbb{B}_{*}$. So condition (2.4) implies that $\bar{w}$ is normal to the tangent space to $\partial \mathbb{B}_{*}$ at $z$. Thus there is a $\varrho=\varrho(z)>0$ for which (2.2) holds.

If $z \bullet z=0$, then (2.4) implies that $\bar{w}$ is normal to the tangent space at $z$ to the $(2 n-3)$-dimensional real manifold $\partial \mathbb{B}_{*} \cap\left\{z \in \mathbb{C}^{n} \backslash\{0\}: z \bullet z=0\right\}$. Thus $\bar{w}$ is in the real span of the vectors $\bar{z}, i \bar{z}$, and $z$. Therefore, there are real numbers $\varrho=\varrho(z), \mu=\mu(z)$, and $v=v(z)$ such that

$$
\bar{w}=(\nu+i \mu) \bar{z}+\varrho z .
$$

Using (2.4) this shows that

$$
\Re((\nu-i \mu)(\zeta \bullet z))+\mathfrak{R}(\varrho(\zeta \bullet \bar{z}))<\varrho \quad \text { for all } \zeta \in \mathbb{B}_{*} .
$$

Taking $\zeta=\lambda \bar{z}$ yields $\Re(\lambda(\nu-i \mu))<\varrho$ for all $\lambda \in \Delta$. This implies that $|\eta| \leq \varrho$, where $\eta:=\nu+i \mu$, and $\varrho>0$ (take $\zeta=0)$.

Lemma 2.3. Let $\varphi=\left(\varphi_{1}, \ldots, \varphi_{n}\right): \Delta \longrightarrow \mathbb{B}_{*}$ be a complex geodesic such that the functions $\varphi \bullet \varphi$ and $\varphi_{j}$ with $j=1, \ldots, n$ do not vanish identically on $\Delta$. Then $\varphi$ is of the form (1.1) and $\varphi \bullet \varphi$ is of the form (1.2).

Proof. By Lemmas 2.1 and 2.2 there are a holomorphic mapping $h=$ $\left(h_{1}, \ldots, h_{n}\right): \Delta \longrightarrow \mathbb{C}^{n}$ with components in the Hardy space $H^{1}(\Delta)$ and a function $\varrho: \partial \Delta \longrightarrow] 0,+\infty[$ such that

$$
\frac{h_{j}^{*}(\lambda)}{\lambda}=\varrho(\lambda)\left[\bar{\varphi}_{j}^{*}(\lambda)+\overline{\frac{\left(\varphi^{*} \bullet \varphi^{*}\right)(\lambda)}{\left|\varphi^{*} \bullet \varphi^{*}\right|(\lambda)}} \varphi_{j}^{*}(\lambda)\right]
$$

almost everywhere on $\partial \Delta$. Since $\frac{\varphi^{*}(\lambda) \bullet h^{*}(\lambda)}{\lambda}=\varrho(\lambda)>0$ for almost all $\lambda \in \Delta$, by [G] there are $r>0$ and $\alpha \in \bar{\Delta}$ such that

$$
(\varphi \bullet h)(\lambda)=r(\lambda-\alpha)(1-\bar{\alpha} \lambda)
$$

on $\Delta$. Without loss of generality we may assume that $r=1$. This implies that $\varrho(\lambda)=|1-\lambda \bar{\alpha}|^{2}$ almost everywhere on $\partial \Delta$ and that each $h_{j}$ is bounded on $\Delta$. So, the product of the functions $h \bullet h$ and $\varphi \bullet \varphi$ is in the Hardy space $H^{1}(\Delta)$ and satisfies the following identity

$$
\frac{\left(h^{*} \bullet h^{*}\right)\left(\varphi^{*} \bullet \varphi^{*}\right)(\lambda)}{\lambda^{2}}=2|1-\lambda \bar{\alpha}|^{4}\left|\varphi^{*}(\lambda) \bullet \varphi^{*}(\lambda)\right|>0
$$


almost everywhere on $\partial \Delta$. By Lemma 18 in [E] there exist $a>0$ and $\beta_{1}, \beta_{2} \in$ $\bar{\Delta}$ such that

$$
\begin{aligned}
(h \bullet h)(\varphi \bullet \varphi)(\lambda) & =a\left(\lambda-\beta_{1}\right)\left(1-\bar{\beta}_{1} \lambda\right)\left(\lambda-\beta_{2}\right)\left(1-\bar{\beta}_{2} \lambda\right) \\
& =\prod_{j \in J} \frac{\lambda-\beta_{j}}{1-\bar{\beta}_{j} \lambda} a \prod_{j \in J}\left(1-\bar{\beta}_{j} \lambda\right)^{2} \prod_{j \notin J}\left(-\beta_{j}\right)\left(1-\bar{\beta}_{j} \lambda\right)^{2}
\end{aligned}
$$

on $\Delta$, where $J:=\left\{j \in\{1,2\}: \beta_{j} \in \Delta\right\}$. Writing $h \bullet h=B S F$ and $\varphi \bullet \varphi=\widetilde{B} \widetilde{S} \widetilde{F}$ where $B, \widetilde{B}$ are Blaschke products, $S, \widetilde{S}$ are singular inner functions and $F, \widetilde{F}$ are singular outer functions, it follows from (2.8) that $S=\widetilde{S}=1$ (see Lemma 19 in $[\mathrm{E}])$. By (2.7) we see that

$$
\begin{aligned}
F(\lambda) & =e^{\frac{1}{2 \pi} \int_{0}^{2 \pi} \frac{e^{i \theta}+\lambda}{e^{i \theta}-\lambda} \log \left|h^{*} \bullet h^{*}\left(e^{i \theta}\right)\right| d \theta} \\
& =e^{\frac{1}{2 \pi} \int_{0}^{2 \pi} \frac{e^{i \theta}+\lambda}{e^{i \theta}-\lambda} \log 2\left|1-e^{i \theta} \bar{\alpha}\right|^{4} d \theta}=2(1-\lambda \bar{\alpha})^{4}, \quad \lambda \in \Delta .
\end{aligned}
$$

Therefore, for some constant $c$,

$$
(B(\varphi \bullet \varphi))(\lambda)=(B \widetilde{B} \widetilde{F})(\lambda)=c \frac{\left(\lambda-\beta_{1}\right)\left(1-\bar{\beta}_{1} \lambda\right)\left(\lambda-\beta_{2}\right)\left(1-\bar{\beta}_{2} \lambda\right)}{(1-\lambda \bar{\alpha})^{4}}, \lambda \in \Delta .
$$

This, combined with (2.8), implies that $\varphi \bullet \varphi$ is of one of the forms given in the statement of the lemma. On the other hand, a little computing shows that for each $j=1, \ldots, n$, we have

$$
\frac{2(\lambda-\alpha)(1-\bar{\alpha} \lambda) h_{j}^{*}(\lambda)-\left(\left(h^{*} \bullet h^{*}\right) \varphi_{j}^{*}\right)(\lambda)}{4(\lambda-\alpha)^{2}(1-\bar{\alpha} \lambda)^{2}} \varphi_{j}^{*}(\lambda)=\frac{1}{2}\left|\varphi_{j}^{*}(\lambda)\right|^{2}>0
$$

almost everywhere on $\partial \Delta$. Using again Lemma 18 in [E] there exist $r_{j}>0$ and $\alpha_{j 1}, \alpha_{j 2} \in \bar{\Delta}$ such that

(2.11) $\left(2(\lambda-\alpha)(1-\bar{\alpha} \lambda) h_{j}(\lambda)-\left(h \bullet h \varphi_{j}\right)(\lambda)\right) \varphi_{j}(\lambda)=r_{j} \prod_{s=1}^{2}\left(\lambda-\alpha_{j s}\right)\left(1-\bar{\alpha}_{j s} \lambda\right)$

on $\Delta$. If we write $\varphi_{j}=B_{j} S_{j} F_{j}$, where $B_{j}, S_{j}$, and $F_{j}$ respectively are a Blaschke product, a singular inner function, and a singular outer function, it follows from (2.10) and (2.11) that $S_{j}=1$ and

$$
F_{j}(\lambda)=\sqrt{\frac{r_{j}}{2}} \prod_{s=1}^{2} \frac{1-\bar{\alpha}_{j s} \lambda}{1-\bar{\alpha} \lambda} .
$$

Since by (2.11) the only possible zeros of $B_{j}$ are the $\alpha_{j s}$ 's, we see that $\varphi_{j}$ has one of the following three forms

$$
a_{j} \frac{\left(1-\bar{\alpha}_{j 1} \lambda\right)\left(1-\bar{\alpha}_{j 2} \lambda\right)}{(1-\bar{\alpha} \lambda)^{2}}, a_{j} \frac{\left(\lambda-\alpha_{j 1}\right)\left(1-\bar{\alpha}_{j 2} \lambda\right)}{(1-\bar{\alpha} \lambda)^{2}}, \text { or } a_{j} \frac{\left(\lambda-\alpha_{j 1}\right)\left(\lambda-\alpha_{j 2}\right)}{(1-\bar{\alpha} \lambda)^{2}}
$$

corresponding to the cases, where $B_{j}$ has no zeros, one zero, or two zeros. Since for all $j=1, \ldots, n$ the function $\varphi_{j}$ is bounded and $\varphi$ is non-constant, it follows that $\alpha \in \Delta$. This completes the proof of the lemma. 
Lemma 2.4. Let $\varphi=\left(\varphi_{1}, \ldots, \varphi_{n}\right): \Delta \longrightarrow \mathbb{B}_{*}$ be a complex geodesic such that $\varphi \bullet \varphi \equiv 0$ and all the functions $\varphi_{j}$ with $j=1, \ldots, n$ do not vanish identically on $\Delta$. Then $\varphi$ is of the form (1.1).

PROOF. Applying Lemmas 2.1 and 2.2 we find a map $h=\left(h_{1}, \ldots, h_{n}\right)$ : $\Delta \longrightarrow \mathbb{C}^{n}$ with components in the Hardy space $H^{1}(\Delta)$ and two functions $\varrho: \partial \Delta \longrightarrow] 0,+\infty[$ and $\eta: \partial \Delta \longrightarrow \mathbb{C}$ such that $|\eta| \leq \varrho$ in $\partial \Delta$ and

$$
\frac{h_{j}^{*}(\lambda)}{\lambda}=\varrho(\lambda) \bar{\varphi}_{j}^{*}(\lambda)+\eta(\lambda) \varphi_{j}^{*}(\lambda)
$$

almost everywhere on $\partial \Delta$. Since $\frac{\varphi^{*}(\lambda) \bullet h^{*}(\lambda)}{\lambda}>0$ for almost all $\lambda \in \partial \Delta$, by [G] there are $r>0$ and $\alpha \in \bar{\Delta}$ such that

$$
(\varphi \bullet h)(\lambda)=r(\lambda-\alpha)(1-\bar{\alpha} \lambda)
$$

on $\Delta$. Without loss of generality we may assume that $r=1$. This implies that $\varrho(\lambda)=|1-\lambda \bar{\alpha}|^{2}$ almost everywhere on $\partial \Delta$. In particular, $\varrho$ and $\eta$ are bounded in $\partial \Delta$. On the other hand a simple calculation gives the following identities

$$
\left\{\begin{aligned}
\frac{\left(h^{*} \bullet h^{*}\right)(\lambda)}{\lambda^{2}} & =2 \varrho(\lambda) \eta(\lambda) \\
\left\|h^{*}(\lambda)\right\|^{2} & =\varrho^{2}(\lambda)+\left|\eta^{2}(\lambda)\right|
\end{aligned}\right.
$$

almost everywhere on $\partial \Delta$. Hence the function $h \bullet h$ belongs to the Hardy space $H^{1}(\Delta)$.

CASE 1: $\eta=0$ on a set of positive measure on $\partial \Delta$.

Then $h \bullet h \equiv 0$ on $\Delta$ and so $\eta=0$ a.e. on $\partial \Delta$. Thus, by (2.13) we see that

$$
\frac{h_{j}^{*}(\lambda)}{\lambda}=\varrho(\lambda) \bar{\varphi}_{j}^{*}(\lambda)
$$

almost everywhere on $\partial \Delta$. Now we observe that $\varphi$ maps $\Delta$ to $\mathbb{B}$ and $\varphi^{*}(\lambda) \in \partial \mathbb{B}$ for almost all $\lambda \in \partial \Delta$, where $\mathbb{B}$ denotes the open unit ball in $\mathbb{C}^{n}$. This, combined with (2.16), implies that $\varphi: \Delta \longrightarrow \mathbb{B}$ is a complex geodesic. Hence, applying [JPZ], [JP], and [E], $\varphi$ is of the form

$$
\varphi_{j}(\lambda)=a_{j}\left(\frac{\lambda-\alpha_{j}}{1-\bar{\alpha}_{j} \lambda}\right)^{k_{j}} \frac{1-\bar{\alpha}_{j} \lambda}{1-\bar{\alpha} \lambda}
$$

for $j=1, \ldots, n$, where $a_{j} \in \mathbb{C}, \alpha \in \Delta, k_{j} \in\{0,1\}$ and $\alpha_{j} \in \bar{\Delta}$ with $\alpha_{j} \in \Delta$ whenever $k_{j}=0$. 
CASE 2: $\eta \neq 0$ a.e. on $\partial \Delta$.

Using (2.13) and (2.15), a little computing shows that

$$
\varphi_{j}^{*}(\lambda) \frac{2 h_{j}^{*}(\lambda)\left(h^{*} \bullet \varphi^{*}\right)(\lambda)-\left(h^{*} \bullet h^{*}\right)(\lambda) \varphi_{j}^{*}(\lambda)}{\lambda^{2}}=2 \varrho^{2}(\lambda)\left|\varphi_{j}^{*}(\lambda)\right|^{2}>0
$$

almost everywhere on $\partial \Delta$. Hence, by Lemma 18 in [E],

$$
\varphi_{j}(\lambda)\left(2 h_{j}(\lambda)(h \bullet \varphi)(\lambda)-(h \bullet h)(\lambda) \varphi_{j}(\lambda)\right)=r_{j} \prod_{s=1}^{2}\left(\lambda-\alpha_{j s}\right)\left(1-\bar{\alpha}_{j s} \lambda\right)
$$

where $\alpha_{j s} \in \bar{\Delta}$ and $r_{j} \in \mathbb{R}, r_{j}>0$. Observe that the only possible zeros of $\varphi_{j}$ are $\alpha_{j 1}$ and $\alpha_{j 2}$. Let $B_{j}, F_{j}$, and $S_{j}$, respectively $\widehat{B}_{j}, \widehat{F}_{j}$, and $\widehat{S}_{j}$, be the Blaschke factor, the outer factor, and the singular inner factor of the function $\varphi_{j}$, respectively $2 h_{j}(h \bullet \varphi)-(h \bullet h) \varphi_{j}$. By (2.17) we see that $S_{j}=\widehat{S}_{j}=1$ and

$$
\begin{aligned}
\left(F_{j} \widehat{F}_{j}\right)(\lambda) & =e^{\frac{1}{2 \pi} \int_{0}^{2 \pi} \frac{e^{i \theta}+\lambda}{e^{i \theta}-\lambda} \log \left(2\left|1-\bar{\alpha} e^{i \theta}\right|^{4}\left|\varphi_{j}^{*}\right|^{2}\left(e^{i \theta}\right)\right) d \theta} \\
& =2(1-\lambda \bar{\alpha})^{4} F_{j}^{2}(\lambda) .
\end{aligned}
$$

Hence,

$$
\widehat{F}_{j}(\lambda)=2(1-\lambda \bar{\alpha})^{4} F_{j}(\lambda)
$$

On the other hand,

$$
\left(F_{j} \widehat{F}_{j}\right)(\lambda)=\tilde{a}_{j} \prod_{s=1}^{2}\left(1-\bar{\alpha}_{j s} \lambda\right)^{2}
$$

with an $\tilde{a}_{j} \in \mathbb{C}$. This implies that

$$
F_{j}(\lambda)=\hat{a}_{j} \prod_{s=1}^{2} \frac{\left(1-\bar{\alpha}_{j s} \lambda\right)}{(1-\lambda \bar{\alpha})}
$$

with an $\hat{a}_{j} \in \mathbb{C}$. Now from (2.18) and (2.19) we obtain that $\varphi$ has the desired form. This completes the proof of the lemma.

The next step will be the proof of the uniqueness of a complex geodesic passing through two different points. First we observe the following simple fact.

Lemma 2.5. Assume that $\varphi: \Delta \rightarrow \mathbb{B}_{*}$ and $\psi: \Delta \rightarrow \mathbb{B}_{*}$ are two geodesics with $\varphi(0)=\psi(0)$ and $\varphi^{\prime}(0)=\psi^{\prime}(0)$. Let $\alpha$ and $\beta$ be the numbers in the denominator of the representation (1.1) of $\varphi$ and $\psi$, respectively. Then $\alpha=\beta$. 
ProOF. We may assume that

$$
\varphi(\lambda)=\frac{P(\lambda)}{(1-\bar{\alpha} \lambda)^{2}} \quad \text { and } \quad \psi(\lambda)=\frac{Q(\lambda)}{(1-\bar{\beta} \lambda)^{2}}, \quad \lambda \in \Delta,
$$

where $P$ and $Q$ are vectors of polynomials of degree $\leq 2$ and $\alpha, \beta \in \Delta$. Then $\frac{1}{2}(\varphi+\psi)$ is again such a geodesic; therefore, in virtue of Theorem A, it can be written as

$$
\frac{1}{2}(\varphi+\psi)(\lambda)=\frac{R(\lambda)}{(1-\bar{\gamma} \lambda)^{2}}, \quad \lambda \in \Delta,
$$

where $R$ is a vector of polynomials of degree $\leq 2$ and $\gamma \in \Delta$. Let us suppose that $\alpha \neq \beta$.

CASE 1: $\gamma=0$.

Then

$$
P(\lambda)(1-\bar{\beta} \lambda)^{2}+Q(\lambda)(1-\bar{\alpha} \lambda)^{2}=2 R(\lambda)(1-\bar{\alpha} \lambda)^{2}(1-\bar{\beta} \lambda)^{2}, \quad \lambda \in \mathbb{C} .
$$

Hence $(1-\bar{\alpha} \cdot)^{2} / P$ and therefore $\varphi$ is identically constant; a contradiction.

CASE 2: $\gamma \neq 0$.

Then for all $\lambda \in \mathbb{C}$ we have

$$
P(\lambda)(1-\bar{\beta} \lambda)^{2}(1-\bar{\gamma} \lambda)^{2}+Q(\lambda)(1-\bar{\alpha} \lambda)^{2}(1-\bar{\gamma} \lambda)^{2}=2 R(\lambda)(1-\bar{\alpha} \lambda)^{2}(1-\bar{\beta} \lambda)^{2} .
$$

Then $\gamma=\alpha$ or $\gamma=\beta$ or $(1-\bar{\gamma} \cdot)^{2} / R$. In the last case this would imply that the geodesic $\frac{1}{2}(\varphi+\psi)$ is constant which is impossible. So we may assume that $\gamma=\alpha$. Division leads to

$$
P(\lambda)(1-\bar{\beta} \lambda)^{2}+Q(\lambda)(1-\bar{\alpha} \lambda)^{2}=2 R(\lambda)(1-\bar{\beta} \lambda)^{2} .
$$

But then $(1-\bar{\beta} \cdot)^{2} / Q$ implies that $\psi$ is a constant; a contradiction.

LEMMA 2.6. A complex geodesic through two different points is uniquely determined.

Proof. Suppose that Lemma 2.6 is not true. Then by Proposition 8.3.2 in [JP] there exist two different geodesics $\varphi: \Delta \longrightarrow \mathbb{B}_{*}$ and $\psi: \Delta \longrightarrow \mathbb{B}_{*}$ with $\varphi(0)=\psi(0)$ and $\varphi^{\prime}(0)=\psi^{\prime}(0)$. Applying that $\mathbb{B}_{*}$ is convex, then also $\chi_{t}:=\varphi+t(\psi-\varphi), t \in[0,1]$, is a geodesic of $\mathbb{B}_{*}$, and therefore a proper map. Hence we have

$$
\left(1-\|(\varphi+t Z)(\lambda)\|^{2}\right)^{2}=|(\varphi+t Z) \bullet(\varphi+t Z)(\lambda)|^{2}, \quad \lambda \in \partial \Delta, t \in[0,1],
$$

where $Z:=\psi-\varphi$. Therefore, exploiting the term in $t^{4}$ we get $\|Z(\lambda)\|^{4}=\mid(Z \bullet$ $Z)\left.(\lambda)\right|^{2}, \lambda \in \partial \Delta$. Write the complex vector $Z=X+i Y$, where $X, Y: \bar{\Delta} \longrightarrow \mathbb{R}^{n}$ are real vector-valued functions. Then $\|X(\lambda)\|\|Y(\lambda)\|=|X(\lambda) \bullet Y(\lambda)|$ meaning that the vectors $X(\lambda)$ and $Y(\lambda)$ are parallel, $\lambda \in \partial \Delta$. By assumption, $Z(\lambda) \neq 0$ 
for all $\lambda \in \partial \Delta$ except finitely many points. Moreover, $Z$ has at least one zero in $\Delta$. Therefore, $\left.X\right|_{\partial \Delta} \not=0$, i.e. $X(\lambda) \neq 0$ for $\lambda$ in a subset $E$ of $\partial \Delta$ of positive measure. Hence $Z(\lambda)=X(\lambda)(1+i \gamma(\lambda)), \lambda \in E$, for suitable real numbers $\gamma(\lambda)$. Assume for a moment we know that

$$
\overline{(\varphi \bullet \varphi)}(\lambda)(Z \bullet Z)(\lambda) \quad \text { is real for all } \lambda \in E \text {. }
$$

According to Lemma 2.5 we may write

$$
\varphi(\lambda)=\frac{P(\lambda)}{(1-\bar{\alpha} \lambda)^{2}} \quad \text { and } \quad \psi(\lambda)=\frac{Q(\lambda)}{(1-\bar{\alpha} \lambda)^{2}}, \quad \lambda \in \Delta,
$$

where $P$ and $Q$ are vectors of polynomials of degree $\leq 2$ and $\alpha \in \Delta$. By our assumption on the geodesics it follows that

$$
P(0)=Q(0) \quad \text { and } P^{\prime}(0)=Q^{\prime}(0)
$$

Therefore, there is a vector $C \in \mathbb{C}^{n}$ such that $(P-Q)(\lambda)=C \lambda^{2}, \lambda \in \mathbb{C}$. Then for $\lambda \in E$ it follows from our previous assumption $(*)$ that

$$
\frac{\overline{(C \bullet C)}(P \bullet P)(\lambda)}{\lambda^{4}|1-\bar{\alpha} \lambda|^{4}}=\frac{(C \bullet C) \lambda^{4} \overline{(P \bullet P)}(\lambda)}{|1-\bar{\alpha} \lambda|^{4}}, \quad \lambda \in E .
$$

Therefore, $\overline{(C \bullet C)}(P \bullet P)(\lambda)=\lambda^{8}(C \bullet C) \overline{(P \bullet P)}(\lambda), \lambda \in E$. Observe that $(C \bullet C) \neq 0$, since for $\lambda \in E$ we have $X(\lambda) \neq 0$; in particular $0 \neq(1+$ $i \gamma(\lambda))^{2}\|X(\lambda)\|^{2}=(Z \bullet Z)(\lambda)=(C \bullet C) \frac{\lambda^{4}}{(1-\bar{\alpha} \lambda)^{4}}$. So , if $P(\lambda)=a_{0}+a_{1} \lambda+a_{2} \lambda^{2}$ and $\widetilde{P}(\lambda)=\bar{a}_{2}+\bar{a}_{1} \lambda+\bar{a}_{0} \lambda^{2}, \lambda \in \mathbb{C}$, with $a_{j} \in \mathbb{C}^{n}, j=0,1,2$, we get

$$
\frac{\overline{(C \bullet C)}}{(C \bullet C)}(P \bullet P)(\lambda)=\lambda^{4}(\widetilde{P} \bullet \widetilde{P})(\lambda), \quad \lambda \in \mathbb{C} .
$$

From this we see that $a_{0} \bullet a_{0}=a_{0} \bullet a_{1}=a_{1} \bullet a_{2}=a_{1} \bullet a_{1}+2 a_{0} \bullet a_{2}=0$ and $\overline{(C \bullet C)}\left(a_{2} \bullet a_{2}\right)=(C \bullet C) \overline{\left(a_{2} \bullet a_{2}\right)}$.

In particular, we have

$$
(\varphi \bullet \varphi)(\lambda)=\frac{\left(a_{2} \bullet a_{2}\right) \lambda^{4}}{(1-\bar{\alpha} \lambda)^{4}}, \quad \lambda \in \Delta
$$

Recall that, if $\varphi \bullet \varphi \not \equiv 0$, then $\varphi \bullet \varphi$ has at most two zeros (counted with multiplicities) in $\Delta$. Therefore, in view of (2.22), we must have $\varphi \bullet \varphi \equiv 0$.

The same argument leads to $\psi \bullet \psi \equiv 0$ and $\frac{\varphi+\psi}{2} \bullet \frac{\varphi+\psi}{2} \equiv 0$ on $\Delta$. Therefore, $\varphi \bullet \psi \equiv 0$ on $\Delta$, which implies that $Z \bullet Z \equiv 0$ on $\Delta$; a contradiction.

Lemma 2.7. Let $\varphi: \Delta \longrightarrow \mathbb{B}_{*}$ be a complex geodesic such that its $j$-th component is not identically zero. Then $\varphi_{j}$ has at most one zero in $\Delta$. 
Proof. Without loss of generality we may assume that $j=n$. Suppose that $\varphi_{n}$ has two zeros. Then there are $\lambda_{j} \in \Delta, j=1,2$, with $\lambda_{1} \neq \lambda_{2}$ and $\varphi_{n}\left(\lambda_{j}\right)=0, j=1,2$, or there is a $\lambda_{0} \in \Delta$ with $\varphi_{n}\left(\lambda_{0}\right)=\varphi_{n}^{\prime}\left(\lambda_{0}\right)=0$.

In the first case we know that $\widetilde{a}_{1}:=\widetilde{\varphi}\left(\lambda_{1}\right) \neq \widetilde{\varphi}\left(\lambda_{2}\right)=$ : $\widetilde{a}_{2}$ with $\widetilde{\varphi}:=$ $\left(\varphi_{1}, \ldots, \varphi_{n-1}\right)$. Then $\widetilde{a}_{j} \in \widetilde{\mathbb{B}_{*}}$, where $\widetilde{\mathbb{B}_{*}}$ is the $(n-1)$-dimensional minimal ball. By Proposition 8.1.15 in [JP] there is a geodesic $\widetilde{\psi}: \Delta \longrightarrow \widetilde{\mathbb{B}_{*}}$ through the points $\widetilde{a}_{j}, j=1,2$. Put $\psi:=(\widetilde{\psi}, 0): \Delta \longrightarrow \mathbb{B}_{*}$. Obviously, $\psi$ is a geodesic in $\mathbb{B}_{*}$ through the points $\varphi\left(\lambda_{j}\right), j=1,2$; a contradiction to Lemma 2.6.

In the second case a similar reasoning also leads to a contradiction.

Proof of Theorem A. The proof of Theorem A follows from the previous lemmas and the fact that, if some of the components of the mapping $\varphi$ is identically zero, then $\varphi$ can be thought as a complex geodesic of a lower dimensional minimal ball obtained by deleting the corresponding components in $\mathbb{C}^{n}$.

\section{3. - Characterization of complex geodesics}

Throughout this section we shall denote by $\mathcal{F}$ the collection of all mappings $\varphi$ of the form (1.1) that satisfy (1.2). For a $\varphi \in \mathcal{F}$ we put

$$
s_{j}:=\bar{\alpha}_{j 1}+\bar{\alpha}_{j 2}, \quad p_{j}:=\bar{\alpha}_{j 1} \bar{\alpha}_{j 2}, \quad j=1, \ldots, n . .
$$

moreover, set $s:=\bar{\beta}_{1}+\bar{\beta}_{2}$ and $p:=\bar{\beta}_{1} \bar{\beta}_{2}$ (here the $\alpha_{j k}$ 's and the $\beta_{k}$ 's are the numbers in representation (1.1) and (1.2) of $\varphi$ ).

Lemma 3.1. Let $\varphi$ be a mapping in $\mathcal{F}$. Then the following properties are equivalent:

(1) $\varphi^{*}(\lambda) \in \partial \mathbb{B}_{*}$ for almost all $\lambda \in \partial \Delta$;

(2) $\varphi$ satisfies the following identities

$$
\left\{\begin{array}{l}
\sum_{j=1}^{n}\left|a_{j}\right|^{2} p_{j}+|b| p=\bar{\alpha}^{2} \\
\sum_{j=1}^{n}\left|a_{j}\right|^{2}\left(1+\left|s_{j}\right|^{2}+\left|p_{j}\right|^{2}\right)+|b|\left(1+|s|^{2}+|p|^{2}\right)=1+4|\alpha|^{2}+|\alpha|^{4} \\
\sum_{j=1}^{n}\left|a_{j}\right|^{2}\left(s_{j}+\bar{s}_{j} p_{j}\right)+|b|(s+\bar{s} p)=2 \bar{\alpha}\left(1+|\alpha|^{2}\right) .
\end{array}\right.
$$


Proof. In view of (1.1) and (1.2) we have for almost all $\lambda \in \partial \Delta$

$$
\begin{aligned}
\left\|\varphi^{*}(\lambda)\right\|^{2} & =\sum_{j=1}^{n}\left|a_{j}\right|^{2} \frac{\left|1-\bar{\alpha}_{j 1} \lambda\right|^{2}\left|1-\bar{\alpha}_{j 2} \lambda\right|^{2}}{|1-\bar{\alpha} \lambda|^{4}} \\
\left|(\varphi \bullet \varphi)^{*}(\lambda)\right| & =|b| \frac{\left|1-\bar{\beta}_{1} \lambda\right|^{2}\left|1-\bar{\beta}_{2} \lambda\right|^{2}}{|1-\bar{\alpha} \lambda|^{4}} .
\end{aligned}
$$

Hence, (1) is true if and only if the following equality holds for almost all $\lambda \in \partial \Delta$, and then for all $\lambda \in \mathbb{C}$,

$$
\begin{aligned}
& \sum_{j=1}^{n}\left|a_{j}\right|^{2}\left(\lambda-\alpha_{j 1}\right)\left(1-\bar{\alpha}_{j 1} \lambda\right)\left(\lambda-\alpha_{j 2}\right)\left(1-\bar{\alpha}_{j 2} \lambda\right) \\
& \quad+|b|\left(\lambda-\beta_{1}\right)\left(1-\bar{\beta}_{1} \lambda\right)\left(\lambda-\beta_{2}\right)\left(1-\bar{\beta}_{2} \lambda\right)=(\lambda-\alpha)^{2}(1-\bar{\alpha} \lambda)^{2} .
\end{aligned}
$$

Identifying the polynomial coefficients of both sides of the latter equality shows finally that (3.1) is equivalent to part (1).

REMARK. Observe that in the description (3.1) we need to know the form of $\varphi \bullet \varphi$ as in (1.2). In fact, we use the $\beta_{j}$ 's in Lemma 3.1.

We also have to describe the elements of $\mathcal{F}$ that are stationary maps. Since here the form of $\varphi \bullet \varphi$ becomes important, we shall discuss the following cases:

CASE 0: $\varphi \bullet \varphi$ has no zeros in $\Delta$, that is, $k_{1}=k_{2}=0$.

CASE 1: $\varphi \bullet \varphi$ has exactly one zero in $\Delta$. Here we may assume, without loss of generality, that $k_{1}=1$ and $k_{2}=0$.

CASE 2: $\varphi \bullet \varphi$ has exactly two zeros in $\Delta$, that is, $k_{1}=k_{2}=1$.

To each of these cases we associate, respectively, one of the holomorphic functions on $\Delta$

$$
\begin{aligned}
& N_{0}(\lambda):=\frac{\bar{b}}{|b|} \frac{(1-\bar{\alpha} \lambda)^{2}\left(\lambda-\beta_{1}\right)\left(\lambda-\beta_{2}\right)}{\left(1-\bar{\beta}_{1} \lambda\right)\left(1-\bar{\beta}_{2} \lambda\right)}, \quad \lambda \in \Delta, \\
& N_{1}(\lambda):=\frac{\bar{b}}{|b|} \frac{(1-\bar{\alpha} \lambda)^{2}\left(\lambda-\beta_{2}\right)}{1-\bar{\beta}_{2} \lambda}, \quad \lambda \in \Delta, \\
& N_{2}(\lambda):=\frac{\bar{b}}{|b|}(1-\bar{\alpha} \lambda)^{2}, \quad \lambda \in \Delta .
\end{aligned}
$$

If $\varphi \in \mathcal{F}$, let $J_{0}(\varphi)$ and $J_{1}(\varphi)$ be the partition of the set $\{1, \ldots, n\}$ such that for $j \in J_{0}(\varphi)$ the component function $\varphi_{j}$ has no zeros in $\Delta$ and for $j \in J_{1}(\varphi)$ the component function $\varphi_{j}$ has exactly one zero in $\Delta$ (we may assume without loss of generality that this zero is $\alpha_{j 1}$ ). Then we have the following 
LeMma 3.2. Let $\varphi$ be a mapping in $\mathcal{F}$ such that $\varphi \bullet \varphi$ does not vanish identically. Then the following properties are equivalent:

(1) $\varphi$ is a stationary map;

(2) there is $l \in\{0,1,2\}$

$$
\begin{cases}\bar{a}_{j}\left(\alpha-\alpha_{j 1}\right)\left(\alpha-\alpha_{j 2}\right)+a_{j} N_{l}(\alpha) \frac{\left(1-\bar{\alpha}_{j 1} \alpha\right)\left(1-\bar{\alpha}_{j 2} \alpha\right)}{\left(1-|\alpha|^{2}\right)^{2}}=0, & j \in J_{0}(\varphi), \\ \bar{a}_{j}\left(1-\bar{\alpha}_{j 1} \alpha\right)\left(\alpha-\alpha_{j 2}\right)+a_{j} N_{l}(\alpha) \frac{\left(\alpha-\alpha_{j 1}\right)\left(1-\bar{\alpha}_{j 2} \alpha\right)}{\left(1-|\alpha|^{2}\right)^{2}}=0, & j \in J_{1}(\varphi) .\end{cases}
$$

Proof. Assume that $\varphi$ is stationary. Then, by the proof of Theorem 2.3, there are a holomorphic mapping $h=\left(h_{1}, \ldots, h_{n}\right): \Delta \longrightarrow \mathbb{C}^{n}$ with components in the Hardy space $H^{1}(\Delta)$ and an $\alpha \in \Delta$ such that

$$
\frac{h_{j}^{*}(\lambda)}{\lambda}=|1-\lambda \bar{\alpha}|^{2}\left[\bar{\varphi}_{j}^{*}(\lambda)+\overline{\frac{\left(\varphi^{*} \bullet \varphi^{*}\right)(\lambda)}{\left|\varphi^{*} \bullet \varphi^{*}\right|(\lambda)}} \varphi_{j}^{*}(\lambda)\right]
$$

almost everywhere on $\partial \Delta$.

If $\varphi \bullet \varphi$ has no zero in $\Delta$, i.e. $k_{1}=k_{2}=0$, then

$$
\frac{\overline{\left(\varphi^{*} \bullet \varphi^{*}\right)(\lambda)}}{\left|\varphi^{*} \bullet \varphi^{*}\right|(\lambda)}=\frac{N_{0}(\lambda)}{(\lambda-\alpha)^{2}}
$$

almost everywhere on $\partial \Delta$.

If $\varphi \bullet \varphi$ has exactly one zero in $\Delta$, and assume without loss of generality that $k_{1}=1$ and $k_{2}=0$, then

$$
\frac{\overline{\left(\varphi^{*} \bullet \varphi^{*}\right)(\lambda)}}{\left|\varphi^{*} \bullet \varphi^{*}\right|(\lambda)}=\frac{N_{1}(\lambda)}{(\lambda-\alpha)^{2}}
$$

almost everywhere on $\partial \Delta$.

Finally, if $\varphi \bullet \varphi$ has two zeros in $\Delta$, then

$$
\frac{\overline{\left(\varphi^{*} \bullet \varphi^{*}\right)(\lambda)}}{\left|\varphi^{*} \bullet \varphi^{*}\right|(\lambda)}=\frac{N_{2}(\lambda)}{(\lambda-\alpha)^{2}}
$$

almost everywhere on $\partial \Delta$. In the first case we have

$$
\begin{aligned}
(\lambda-\alpha) h_{j}^{*}(\lambda) & =\lambda(\lambda-\alpha)|1-\lambda \bar{\alpha}|^{2}\left[\bar{\varphi}_{j}^{*}(\lambda)+\frac{N_{0}(\lambda)}{(\lambda-\alpha)^{2}} \varphi_{j}^{*}(\lambda)\right] \\
& =(\lambda-\alpha)^{2}(1-\lambda \bar{\alpha})\left[\bar{\varphi}_{j}^{*}(\lambda)+\frac{N_{0}(\lambda)}{(\lambda-\alpha)^{2}} \varphi_{j}^{*}(\lambda)\right] \\
& =(1-\lambda \bar{\alpha})\left[\bar{a}_{j}\left(\lambda-\alpha_{j 1}\right)\left(\lambda-\alpha_{j 2}\right)+a_{j} N_{0}(\lambda) \frac{\left(1-\bar{\alpha}_{j 1} \lambda\right)\left(1-\bar{\alpha}_{j 2} \lambda\right)}{(1-\bar{\alpha} \lambda)^{2}}\right]
\end{aligned}
$$


almost everywhere on $\partial \Delta$. This implies that

$$
(\lambda-\alpha) h_{j}(\lambda)=(1-\lambda \bar{\alpha})\left[\bar{a}_{j}\left(\lambda-\alpha_{j 1}\right)\left(\lambda-\alpha_{j 2}\right)+a_{j} N_{0}(\lambda) \frac{\left(1-\bar{\alpha}_{j 1} \lambda\right)\left(1-\bar{\alpha}_{j 2} \lambda\right)}{(1-\bar{\alpha} \lambda)^{2}}\right]
$$

for all $\lambda \in \Delta$. Hence we have the first equality in (3.2). The two remaining equalities can be proved similarly. To prove the converse, we only consider the first case, the other cases can be checked in a similar way. Assume that (3.2) holds and let $j$ satisfy the first equality in (3.2). Then the function

$$
h_{j}(\lambda):=\frac{1-\lambda \bar{\alpha}}{\lambda-\alpha}\left[\bar{a}_{j}\left(\lambda-\alpha_{j 1}\right)\left(\lambda-\alpha_{j 2}\right)+a_{j} N_{0}(\lambda) \frac{\left(1-\bar{\alpha}_{j 1} \lambda\right)\left(1-\bar{\alpha}_{j 2} \lambda\right)}{(1-\bar{\alpha} \lambda)^{2}}\right]
$$

is holomorphic on the unit disc and belongs to the Hardy space $H^{1}(\Delta)$. In addition its boundary values verify (3.3).

Combining Lemma 3.1 and Lemma 3.2 we obtain

Proposition 3.3. Let $\varphi$ be a mapping in $\mathcal{F}$ such that $\varphi \bullet \varphi$ does not vanish identically. Then $\varphi$ is a complex geodesic if and only if $\varphi$ satisfies (3.1) and (3.2).

REMARK. We should point out that the above characterization involves the shape (1.2) of $\varphi \bullet \varphi$.

Now we discuss the case where $\varphi \bullet \varphi$ vanishes identically. Let $\varphi=$ $\left(\varphi_{1}, \ldots, \varphi_{n}\right)$ be a mapping of the form (1.1). Then we define

$$
N_{j}(\lambda):=(1-\bar{\alpha} \lambda)^{2} \varphi_{j}(\lambda), \quad \lambda \in \Delta .
$$

This is a complex polynomial $\sum_{k=0}^{2} d_{j k} \lambda^{k}$. We set

$$
\widetilde{N}_{j}(\lambda):=\sum_{k=0}^{2} \bar{d}_{j k} \lambda^{2-k}, \quad \lambda \in \Delta .
$$

Observe that we have $(\lambda-\alpha)^{2} \bar{\varphi}_{j}(\lambda)=\widetilde{N}_{j}(\lambda), \lambda \in \partial \Delta$.

Proposition 3.4. Let $\varphi$ be a non-zero mapping of the form (1.1) such that $\varphi \bullet \varphi \equiv 0$. Then $\varphi$ is stationary if and only if there is a $\gamma \in \bar{\Delta}$ such that $\widetilde{N}_{j}(\alpha)=$ $\gamma N_{j}(\alpha)$ for all $j=1, \ldots, n$.

Proof. Assume that $\varphi$ is stationary (for details, see the proof of Lemma 2.4). Then there are functions $h_{j} \in H^{1}(\Delta), j=1, \ldots, n$, such that

$$
h_{j}^{*}(\lambda)=\frac{1-\bar{\alpha}}{\lambda-\alpha}\left(\widetilde{N}_{j}(\lambda)+\lambda(\lambda-\alpha) \eta(\lambda) \frac{N_{j}(\lambda)}{(1-\bar{\alpha} \lambda)^{3}}\right), \quad \lambda \in \partial \Delta .
$$


Recall that $(h \bullet h)(\lambda)=2 \varrho(\lambda) \lambda^{2}, \lambda \in \partial \Delta$. Therefore,

$$
h_{j}^{*}(\lambda)=\frac{1-\bar{\alpha} \lambda}{\lambda-\alpha}\left(\tilde{N}_{j}(\lambda)+\frac{(h \bullet h)(\lambda) N_{j}(\lambda)}{2(1-\bar{\alpha} \lambda)^{4}}\right), \quad \lambda \in \partial \Delta .
$$

Hence we get

$$
\frac{\lambda-\alpha}{1-\bar{\alpha} \lambda} h_{j}(\lambda)=\widetilde{N}_{j}(\lambda)+\frac{(h \bullet h)(\lambda)}{2(1-\bar{\alpha} \lambda)^{4}} N_{j}(\lambda), \quad \lambda \in \Delta ;
$$

in particular,

$$
0=\widetilde{N}_{j}(\alpha)+\frac{(h \bullet h)(\alpha)}{2\left(1-|\alpha|^{2}\right)^{4}} N_{j}(\alpha)
$$

Moreover,

$$
\left|\tilde{N}_{j}(\alpha)\right|=\frac{\mid h \bullet h)(\alpha) \mid}{2\left(1-|\alpha|^{2}\right)^{4}}\left|N_{j}(\alpha)\right| .
$$

Using that $|(h \bullet h)| \leq 2 \varrho|\eta| \leq 2 \varrho^{2}$ almost everywhere on $\partial \Delta$, the maximum principle leads to $\left|\tilde{N}_{j}(\alpha)\right| \leq\left|N_{j}(\alpha)\right|$. Therefore, $\gamma \in \partial \Delta$, which finishes the proof of the "if" part.

Assume now that the condition on the right-hand side in Proposition 3.4 is satisfied. Define

$$
h_{j}(\lambda):=\frac{1-\bar{\alpha} \lambda}{\lambda-\alpha}\left(\tilde{N}_{j}(\lambda)-\gamma N_{j}(\lambda)\right), \quad \lambda \in \Delta, j=1, \ldots, n
$$

Then $h_{j} \in H^{1}(\Delta)$ and

$$
\frac{h_{j}(\lambda)}{\lambda}=|1-\bar{\alpha} \lambda|^{2} \bar{\varphi}_{j}(\lambda)-\gamma \frac{(1-\bar{\alpha} \lambda)^{3}}{\lambda(\lambda-\alpha)} \varphi_{j}(\lambda), \quad \lambda \in \partial \Delta .
$$

Setting $\eta(\lambda):=-\gamma \frac{(1-\bar{\alpha} \lambda)^{3}}{\lambda(\lambda-\alpha)}, \lambda \in \partial \Delta$, we see that $|\eta(\lambda)| \leq|1-\bar{\alpha} \lambda|^{2}=: \varrho(\lambda)$, $\lambda \in \partial \Delta$ Hence, $\varphi$ is a stationary map.

REMARK. Observe that a geodesic $\varphi$ for $\mathbb{B}_{*}$ with $\varphi \bullet \varphi \equiv 0$ is always a proper map to the unit ball $\mathbb{B}$; in virtue of the proof of Proposition 3.4 , it is also a geodesic for $\mathbb{B}$ iff $\gamma$ can be chosen to be 0 .

It would be of interest to find an effective formula for the Kobayashi distance exploiting the form of the complex geodesics given above. 


\section{REFERENCES}

[E] A. Edigarian, On extremal mappings in complex ellipsoids, Ann. Polon. Math. 62 (1995), 83-96.

[G] G. GentiLi, Regular complex geodesics in the domain $D_{n}=\left\{\left(z_{1}, \ldots, z_{n}\right) \in \mathbb{C}^{n}\right.$ : $\left.\left|z_{1}\right|+\cdots+\left|z_{n}\right|<1\right\}$, In: "Complex Analysis III", C. A. Berenstein (ed.), Lecture Notes in Math. Vol. 1275, Springer-Verlag, Berlin, 1987, pp. 235-252.

[HP] K. T. HAHn - P. Pflug, On a minimal complex norm that extends the real Euclidean norm, Monatsh. Math. 105 (1988), 107-112.

[JP] M. JARnicki - P. Pflug, "Invariant Distances and Metrics in Complex Analysis", de Gruyter Expositions in Mathematics, Walter de Gruyter, 1993.

[K] K. T. KIM, Automorphism group of certain domains with singular boundary, Pacific J. Math. 51 (1991), 54-64.

[MY] G. Mengotti - E. H. Youssfi, The weighted Bergman projection and related theory on the minimal ball, Bull. Sci. Math. 123 (1999), 501-525.

[OPY] K. Oeljeklaus - P. Pflug - E. H. Youssfi, The Bergman kernel of the minimal ball and applications, Ann. Inst. Fourier (Grenoble) 47 (1997), 915-928.

[OY] K. Oeljeklaus - E. H. Youssfi, Proper holomorphic mappings and related automorphism groups, J. Geom. Anal. 7 (1997), 623-636.

[PY] P. Pflug - E. H. Youssfi, The Lu Qi-Keng conjecture fails for strongly convex algebraic domains, Arch. Math. 71 (1998), 240-245.

[Z] W. Zwonek, Automorphism group of some special domain in $\mathbb{C}^{n}$, Univ. Iagel. Acta Math. 33 (1996), 185-189.

Institut für Mathematik

Postfach 2503

Universität Oldenburg

26111 Oldenburg, Germany

pflug@mathematik.uni-oldenburg.de

LATP, U.M.R. C.N.R.S. 6632

CMI, Université de Provence

39 Rue F-Joliot-Curie

13453 Marseille Cedex 13, France

youssfi@gyptis.univ-mrs.fr. 